\title{
Glomerular expression of fractalkine is induced by polyinosinic-polycytidylic acid in human mesangial cells: possible involvement of fractalkine after viral infection
}

\author{
Tomomi Aizawa-Yashiro', Tadaatsu Imaizumi², Kazushi Tsuruga', Shojiro Watanabe' ', Tomoh Matsumiya², Ryo Hayakari², \\ Hidemi Yoshida' ${ }^{2}$ Kei Satoh², Etsuro Ito' and Hiroshi Tanaka ${ }^{1,3}$
}

BACKGROUND: Viral infections often trigger the onset or worsening of glomerular diseases, but the details of this mechanism are unclear. Fractalkine/CX3CL1 (Fkn) is a chemokine that induces the chemotaxis and activation of cells expressing its receptor, CX3CR1. To examine the involvement of glomerular Fkn expression in the development of glomerulonephritis after viral infection, we conducted experimental studies using human mesangial cells (MCs) in culture.

METHODS: We examined the effect of polyinosinic-polycytidylic acid (poly IC), an authentic viral double-stranded RNA, on Fkn expression in MCs to investigate the involvement of Fkn in the antiviral reaction of MCs. Fkn mRNA and protein were analyzed using real-time PCR and enzyme-linked immunosorbent assay. Also, an immunofluorescent study to examine mesangial Fkn expression in biopsy specimens obtained from patients with glomerulonephritis was conducted.

RESULTS: Poly IC-induced Fkn expression in MCs in both a time- and dose-dependent manner, and RNA interference (RNAi) against Toll-like receptor 3 (TLR3) or interferon regulatory factor 3 (IRF3) inhibited poly IC-induced Fkn expression. Significant glomerular Fkn expression was observed in biopsy specimens from patients with immunoglobulin A nephropathy and purpura nephritis, with increasing severity of glomerular inflammation.

CONCLUSION: The TLR3/IRF3/Fkn signaling pathway may, at least in part, mediate immune and inflammatory responses against viral infection in MCs.

G lomerular expression of chemokines and chemokine-induced infiltration of inflammatory cells expressing their receptors in the mesangial area are important pathological features of the acute phase of glomerulonephritis (1-3). Fractalkine/CX3CL1 (Fkn) is a unique chemokine that exists in either a membrane-bound or soluble form. Membrane-bound Fkn acts as an adhesion molecule, and the soluble form has potent chemoattractant activity for inflammatory cells expressing its receptor, CX3CR1 (4). Fkn expression is primarily regulated by proinflammatory cytokines such as tumor necrosis factor- $\alpha$ (TNF- $\alpha$ ) and interleukin (IL)-1 (4-6). Fkn expression in mesangial lesions has been reported to be significantly correlated with histopathological disease activity in a rat model of prolonged proliferative glomerulonephritis (7) and in human and mouse models of lupus nephritis (LN) $(8,9)$. Moreover, mesangial Fkn expression may play an important role in the development of prolonged glomerular inflammation (7), and an antagonist of Fkn ameliorates the progression of this condition in a mouse model of LN $(9,10)$. In addition, in CX3CR1positive leukocytes, such as activated monocytes, infiltration was observed in glomerular and tubulointestitial lesions in human glomerulonephritis models $(8,11)$. Thus, both glomerular Fkn expression and recruitment of inflammatory cells expressing the receptor for Fkn may contribute to the development of glomerular inflammation.

By contrast, it is well known that viral infections often trigger the development of inflammatory renal diseases or aggravate preexisting glomerulonephritis (12). Polyinosinic-polycytidylic acid (poly IC) is an authentic double-stranded RNA and a potent inducer of type I interferons (IFNs) and downstream molecules involved in the innate immune pathway. Thus, poly IC has been widely used to mimic viral infection in various cell types such as cultured mesangial cells (MCs) (13-15). Toll-like receptor 3 (TLR3), retinoic acid-inducible gene-I (RIG-I), and melanoma differentiation antigen 5 (MDA5) are reported to serve as receptors for both viral double-stranded RNA and poly IC, and treatment with poly IC induces the expression of type I IFNs and various chemokines, even in MCs (13-17). However, the role of Fkn in innate immune response against virus infection in MCs remains to be elucidated. This study was conducted to evaluate the potential role of Fkn in response to poly IC in cultured normal human MCs. We used immunofluorescence to examine mesangial Fkn expression in biopsy specimens obtained from patients with various renal diseases, including inflammatory and noninflammatory glomerular diseases.

\section{RESULTS}

Poly IC Induces Fkn Expression in MCs

Poly IC treatment of MCs induced Fkn expression in a timedependent manner. Stimulation with poly IC elevated the expression of both Fkn mRNA and protein, reaching maximal 
expression levels at 4 and $24 \mathrm{~h}$ poststimulation, respectively (Figure 1a,b). In contrast, induction of Fkn mRNA expression by poly IC/cationic lipid complex treatment was minimal, whereas RIG-I mRNA and protein were significantly induced (Figure 1c-e). Concerning levels of the soluble and membranebound forms of Fkn, concentration of Fkn was measured in the conditioned medium and in cell lysates of MCs treated with poly IC or poly IC/cationic lipid complex for $24 \mathrm{~h}$. Only
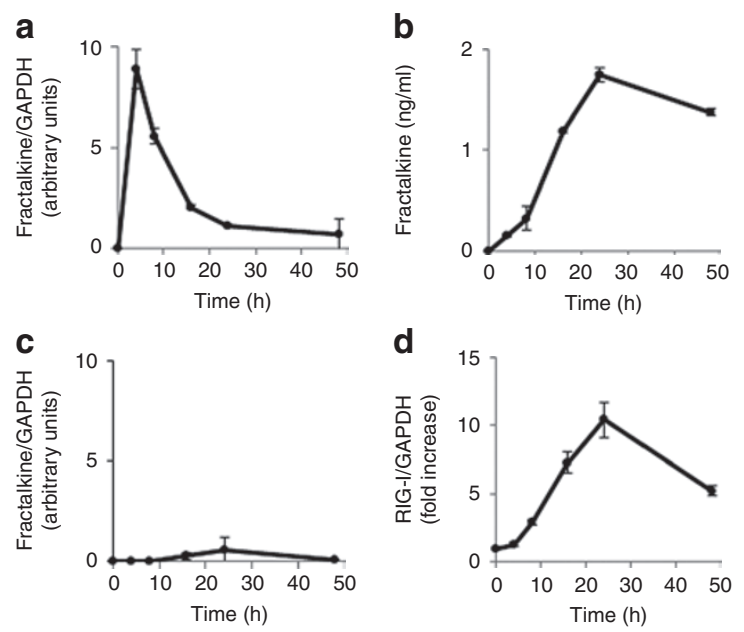
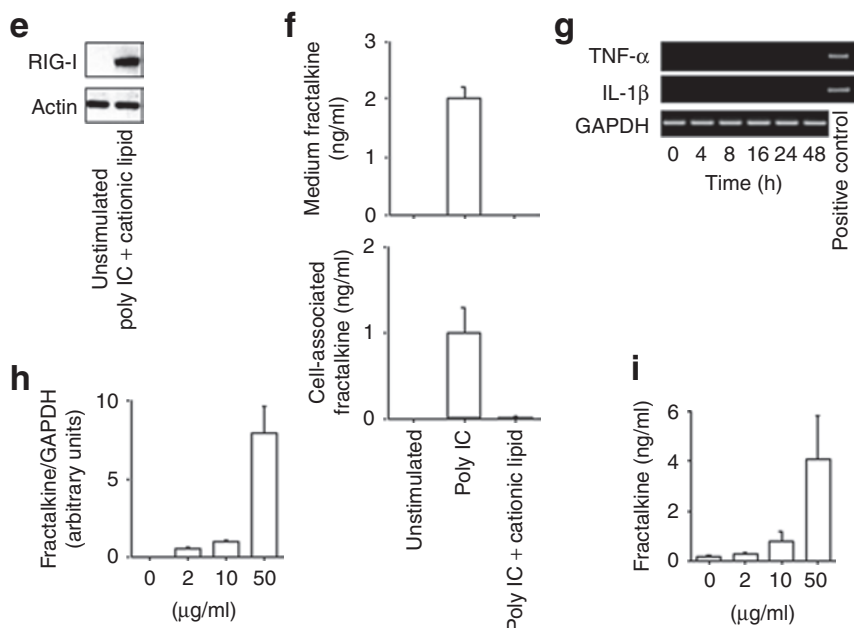

Figure 1. Poly IC induces the expression of Fkn in MCs in a time- and concentration-dependent manner. The cells were treated with $30 \mu \mathrm{g} / \mathrm{ml}$ poly IC for up to $48 \mathrm{~h}$. Conditioned medium was collected and RNA was extracted from the cells. (a) Real-time quantitative PCR and (b) enzyme-linked immunosorbent assay analyses were performed. In MCs transfected with poly IC as a complex with cationic lipid (poly IC/cationic lipid), (c) the expression of Fkn mRNA was minimal whereas (d,e) RIG-I mRNA and protein were significantly increased. (f) Fkn protein levels in the conditioned medium (upper panel) and cell lysates (lower panel) from MCs $24 \mathrm{~h}$ after incubation with poly IC or poly IC/cationic lipid. The data are shown as the mean \pm SD $(n=$ 3). (g) Furthermore, RT-PCR for TNF- $\alpha$ and IL-1 $\beta$ were performed as shown. Complementary DNA from blood mononuclear cells was used as a positive control. The cells were treated with various concentrations of poly IC. (h) After $4 \mathrm{~h}$ of incubation, RNA was extracted and subjected to real-time PCR analysis. (i) The medium was collected after $16 \mathrm{~h}$ of incubation, and the concentration of Fkn was measured. Fkn, fractalkine; GAPDH, glyceraldehyde 3-phosphate dehydrogenase; IL, interleukin; MC, mesangial cell; poly IC, polyinosinic-polycytidylic acid; RIG-I, retinoic acid-inducible gene-I; RT-PCR, reverse transcription-PCR; TNF, tumor necrosis factor. poly IC treatment increased both secreted and cell-associated Fkn protein levels (Figure 1f). By contrast, treatment of cells with poly IC did not induce the expression of TNF- $\alpha$ or IL- $1 \beta$ mRNA (Figure 1g).

Expression of Fkn mRNA and protein in a concentrationdependent manner was also observed after stimulation of poly IC (Figure 1h,i).

\section{TLR3 Is Involved in Poly IC-Induced Fkn Expression}

Poly IC-induced Fkn mRNA and protein expressions were suppressed in response to treatment with small-interfering RNA (siRNA) against TLR3, whereas siRNAs against RIG-I and MDA5 did not affect poly IC-induced Fkn mRNA expression (Figure 2a,b). Effective knockdown of each target gene by the appropriate siRNA was confirmed using western blot analyses (Figure 2c).

\section{Interferon Regulatory Factor 3 siRNA Suppressed Poly IC-Induced Fkn Expression}

Transfection of MCs with siRNA against interferon regulatory factor 3 (IRF3) suppressed the expressions of Fkn mRNA and protein (Figure 3a,b). By contrast, siRNA against nuclear factor (NF)- $\mathrm{kB}$ p65 did not affect Fkn protein expression, whereas this treatment clearly decreased IL-8 protein expression (Figure 3c). Effective knockdown of IRF3 and NF- $\kappa$ B p65 by siRNAs were confirmed by western blot analyses (Figure 3d). Furthermore, we examined the effect of RNA interference (RNAi) against TLR3 and IFR3 on the expression
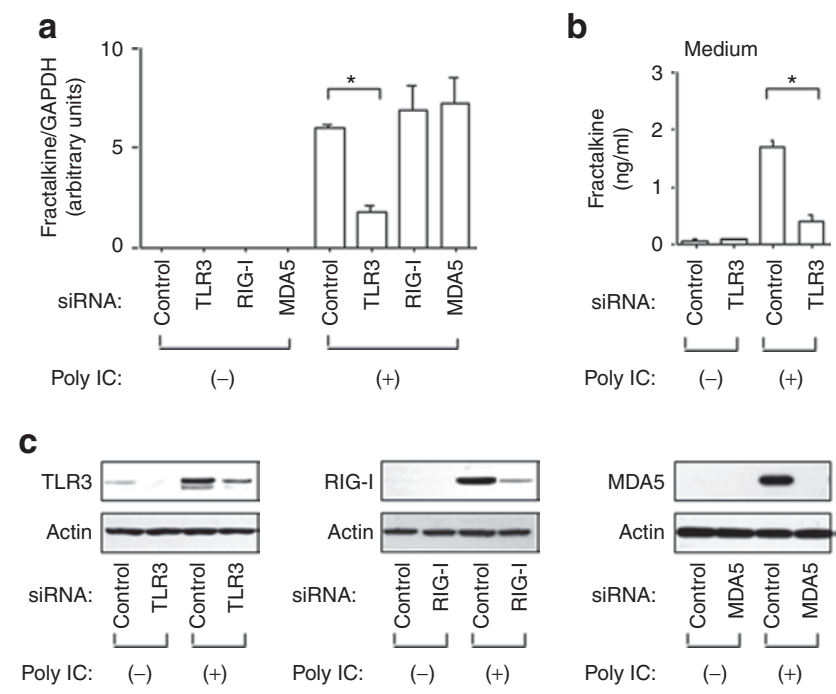

Figure 2. Knockdown of TLR3 inhibits the poly IC-induced expression of Fkn in MCs. The cells were transfected with siRNAs against TLR3, RIG-I, and MDA5 or control siRNA. (a) After $24 \mathrm{~h}$ of transfection, the cells were treated with $30 \mu \mathrm{g} / \mathrm{ml}$ poly IC. RNA was extracted from the cells after $4 \mathrm{~h}$ of incubation, and quantitative real-time PCR was performed $(n=3, * P$ $<0.01)$. Conditioned medium and protein were collected after $16 \mathrm{~h}$ of incubation. (b) Fkn in the medium was measured using an enzyme-linked immunosorbent assay $\left(n=3,{ }^{*} P<0.01\right)$. (c) The knockdown of TLR3, RIG-I, and MDA5 was confirmed using western blot analyses. Fkn, fractalkine; MC, mesangial cell; MDA5, melanoma differentiation antigen 5; poly IC, polyinosinic-polycytidylic acid; RIG-I, retinoic acid-inducible gene-I; siRNA, small-interfering RNA; TLR3, Toll-like receptor 3. 
a

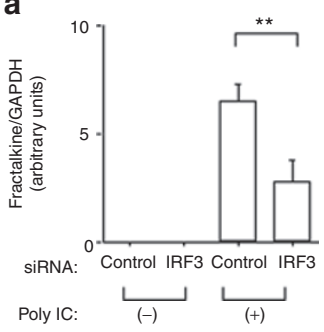

b
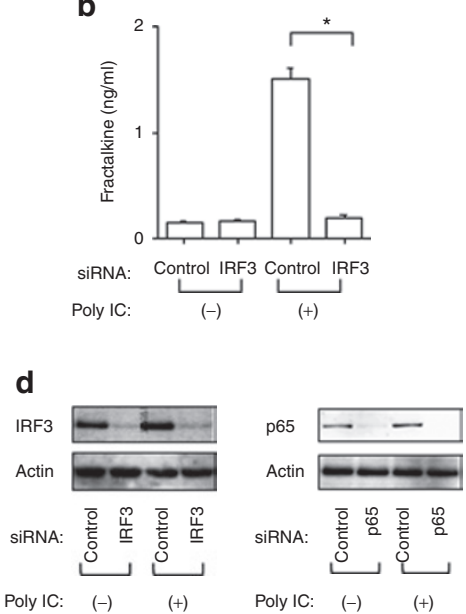

C
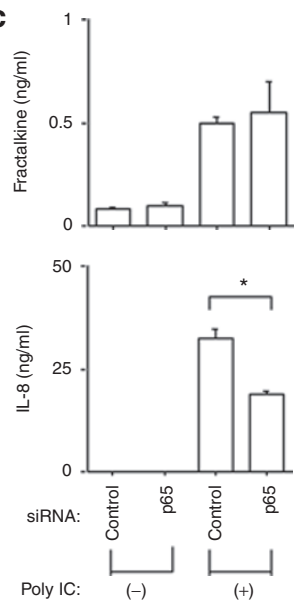

e

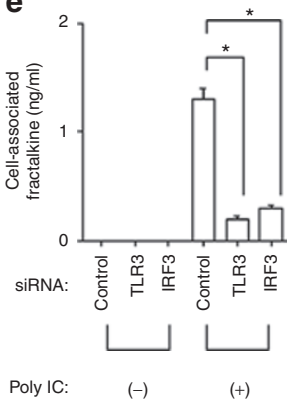

Figure 3. Knockdown of IRF3, but not NF-KB, inhibits poly IC-induced expression of Fkn in MCs. Cells were transfected with siRNA against IRF3, NF-KB p65, or control siRNA. After $24 \mathrm{~h}$ of transfection, the cells were treated with $30 \mu \mathrm{g} / \mathrm{ml}$ poly IC. RNA, protein, the conditioned medium, and cell lysates were collected and subjected to (a) quantitative real-time PCR, (b,c,e) enzyme-linked immunosorbent assay, and (d) western blot $\left(n=3,{ }^{*} P<0.01,{ }^{* *} P<0.05\right)$. Fkn, fractalkine; GAPDH, glyceraldehyde 3-phosphate dehydrogenase; IL, interleukin; IRF3, interferon regulatory factor 3; MC, mesangial cell; NF-KB, nuclear factor- $\mathrm{KB}$; poly IC, polyinosinicpolycytidylic acid; siRNA, small-interfering RNA; TLR3, Toll-like receptor 3 .

level of cell-associated Fkn protein using poly IC-treated cell lysates. Knockdown of TLR3 or IFR3 clearly decreased poly IC-induced cell-associated Fkn protein (Figure 3e).

The Effect of an Activation Protein-1 Inhibitor on Poly IC-Induced Fkn Expression

Pretreatment of cells with the activation protein-1 (AP-1) inhibitor SR11302 did not affect the induction of Fkn mRNA expression by poly IC (Figure 4 ).

\section{The Effect of an Anti-IFN Receptor Antibody on Poly IC-Induced Fkn Expression}

Pretreatment of cells using a blocking antibody against type I IFN receptor did not affect poly IC-induced Fkn mRNA expression, although this treatment clearly suppressed mRNA expression of poly IC-induced interferon-stimulated gene (ISG) 15 (Figure 5a,b).

\section{Immunofluorescence Staining of Fkn}

Significant positive staining of Fkn in the mesangial area was observed in the biopsy specimen of LN (Figure 6a) as

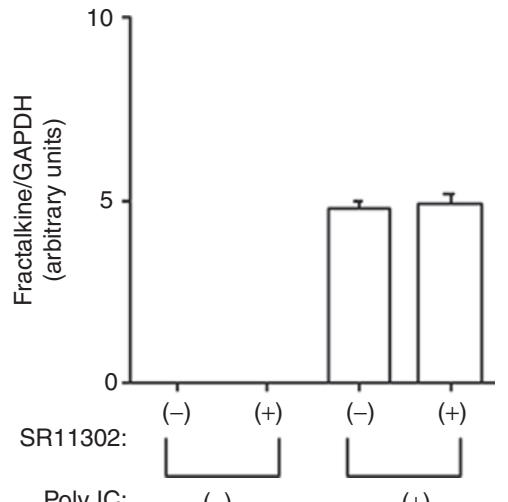

$(-)$

$(+)$

Figure 4. SR11302, an AP-1 inhibitor, did not affect the poly-IC-induced Fkn mRNA expression in MCs. Cells were pretreated with $5 \mu \mathrm{mol} / \mathrm{I} \mathrm{SR} 11302$ $1 \mathrm{~h}$ before the treatment with poly IC. After incubating for $4 \mathrm{~h}$, RNA was extracted and real-time RT-PCR was performed. AP-1, activation protein-1; Fkn, fractalkine; GAPDH, glyceraldehyde 3-phosphate dehydrogenase; MC, mesangial cell; poly IC, polyinosinic-polycytidylic acid; RT-PCR, reverse transcription-PCR. a

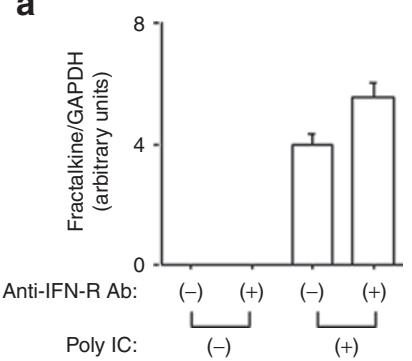

b

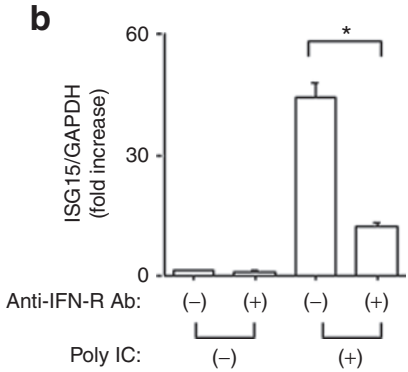

Figure 5. Effect of an anti-interferon-receptor antibody (anti-IFN-R Ab) on the expression of poly IC-induced Fkn and ISG15 mRNA. After $1 \mathrm{~h}$ of pretreatment with an anti-IFN-R Ab, the cells were treated with $30 \mu \mathrm{g} /$ $\mathrm{ml}$ poly IC for $4 \mathrm{~h}$. $(\mathbf{a}, \mathbf{b})$ RNA was collected and quantitative real-time PCR was performed $\left(n=3,{ }^{*} P<0.01\right)$. Fkn, fractalkine; GAPDH, glyceraldehyde 3-phosphate dehydrogenase; ISG15, interferon-stimulated gene 15; poly IC, polyinosinic-polycytidylic acid.

previously reported (8). Of note, significant Fkn staining was also observed in the mesangial area of active immunoglobulin A nephropathy (IgAN) with high activity index (AI) and chronicity index (CI) (Figure 6d,e), but this was not detected in a renal biopsy specimen of a patient with IgAN having low AI and CI (Figure 6f). Moreover, Fkn was markedly expressed in the mesangial area of crescentic Henoch-Schönlein purpura nephritis (HSPN) (International Study of Kidney Disease in Children (ISKDC) grade IIIb and IIIa) (Figure $5 \mathrm{~g}$,h), but this was not observed for noncrescentic HSPN (ISKDC grade II). In cases of nonproliferative glomerular diseases-minimalchange nephrotic syndrome and noninflammatory glomerular lesion (nutcracker syndrome) - glomerular Fkn expression was not detected in biopsy specimens (Figure 6b,c). However, except for histologic severity, there were no correlations between clinical parameters, such as the degrees of proteinuria and hematuria, and renal function, and an increased intensity of Fkn immunostaining in the biopsy specimens of the patients was observed because few patients with a normal renal function 

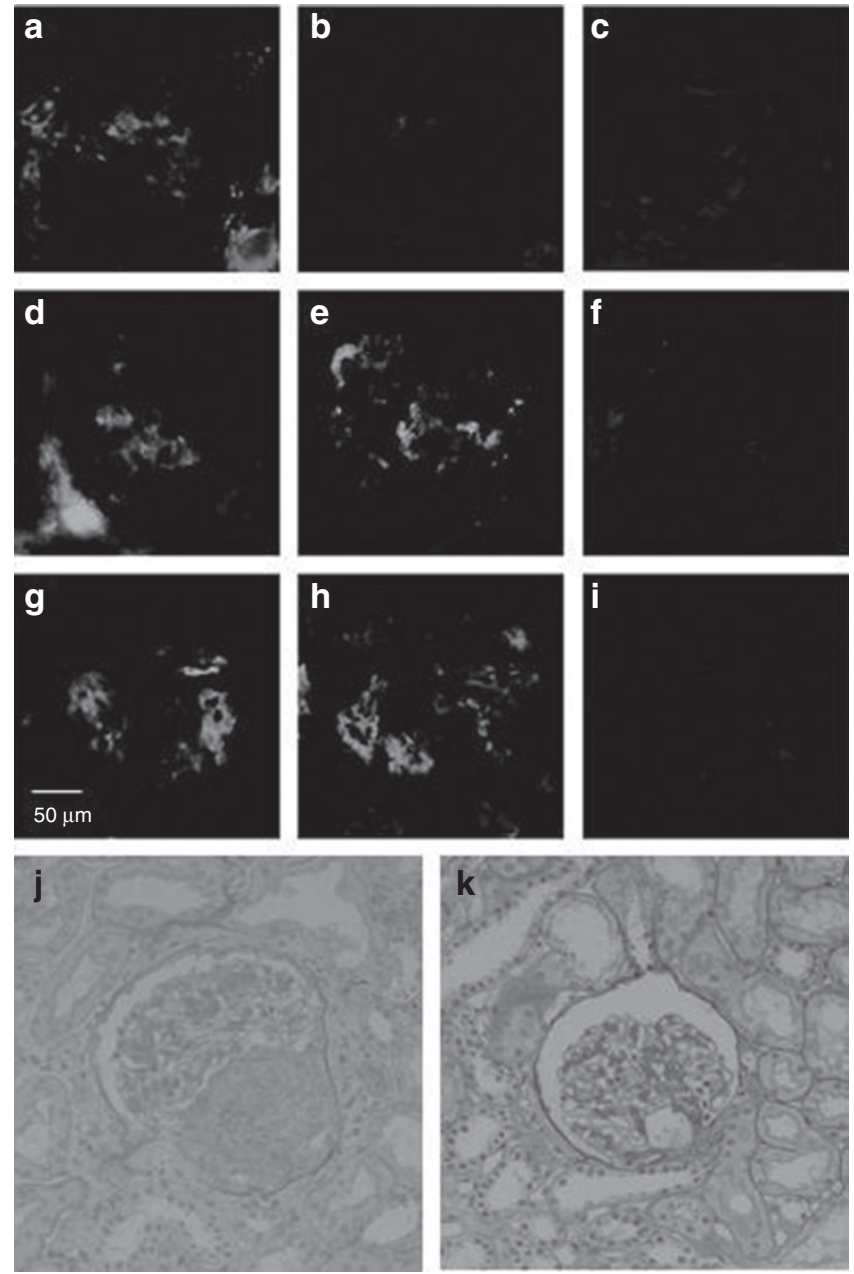

Figure 6. Immunofluorescence reactivity for Fkn in renal biopsy specimens from patients with (a) lupus nephritis (LN, served as a positive control), (b) minimal-change nephrotic syndrome (MCNS), (c) nutcracker syndrome (served as a noninflammatory glomerular disease), (d-f) IgA nephropathy ( $\operatorname{lgAN})$, and (g-i) Henoch-Schönlein purpura nephritis (HSPN) (a bar of $50 \mu \mathrm{m}$ applies to $\mathbf{a}-\mathbf{i}$, original magnification, $\times 200)$. Specimens were collected from patients with IgAN with a high activity index (Al) and chronicity index $(\mathrm{Cl})$ as shown in $\mathbf{d}$ and $\mathbf{e}$, and a specimen was collected from a patient with a low $\mathrm{Al}$ and $\mathrm{Cl}$ as shown in $\mathbf{f}$. Specimens of the International Study of Kidney Disease of Children (ISKDC) grades IIIb, IIla, and II were collected from patients with HSPN (g, h, and $\mathbf{i}$, respectively). A significant increase in Fkn immunoreactivity was observed in patients with IgAN with high Al and $\mathrm{Cl}(\mathbf{d}, \mathbf{e})$, and in patients with ISKDC grade IIIb and IIIa HSPN (g,h), as well as in a patient with LN (a). By contrast, glomerular immunoreactivity for Fkn was negligible in biopsy specimens from patients with MCNS, nutcracker syndrome, IgAN with low Al and $\mathrm{Cl}(\mathbf{f})$, and ISKDC grade II HSPN (i). Light microscopic images in patients with high $\mathrm{Al}$ and $\mathrm{Cl}(\mathbf{j})$ and low $\mathrm{Al}$ and $\mathrm{Cl}$ (k) are shown (periodic acid-Schiff staining, original magnification, $\times 100$ ). Specimens in $\mathbf{j}$ and $\mathbf{k}$ were obtained from the same patients as those in $\mathbf{d}$ and $\mathbf{f}$, respectively. Fkn, fractalkine.

were examined. Typical light microscopy images in patients with IgAN with high AI and CI, and IgAN with low $\mathrm{AI}$ and $\mathrm{CI}$ are represented in Figure $6 \mathrm{j}, \mathrm{k}$.

\section{DISCUSSION}

Fkn was originally discovered in vascular endothelial cells activated by TNF- $\alpha$ or IL-1 (4). Fkn expression is reportedly induced by TNF- $\alpha$ or connective tissue growth factor in MCs; in addition, mesangial expression of Fkn is thought to play a role in the pathogenesis of inflammatory glomerulonephritis $(18,19)$. In a rat model of experimental proliferative glomerulonephritis, marked glomerular expression of Fkn and the recruitment of inflammatory cells expressing its receptor, CX3CR1, were associated with prolonged inflammatory glomerular lesions, but this was not observed with reversible inflammatory lesions (7). Furthermore, in a mouse model of $\mathrm{LN}$, it has been reported that significant glomerular expression of Fkn and $\mathrm{CD} 16^{+}$monocyte infiltration were detected in severe inflammatory lesions; of note, Fkn antagonist administration showed a protective effect against proliferative LN (9). Furthermore, disease activity and proliferative glomerular LN lesions were associated with glomerular Fkn expression and $\mathrm{CD} 16^{+}$monocyte infiltration in patients with LN (8). These experimental and clinical observations suggest that glomerular Fkn expression contributes, at least in part, to the development of proliferative glomerulonephritis, including proliferative LN. However, the details regarding the glomerular signaling pathways involved in Fkn expression remain to be elucidated.

In a proportion of patients with IgAN, RNA viral infections often aggravate preexisting glomerular inflammation (12). Thus, we examined a possible role of glomerular expression of Fkn in the aggravation of IgAN and HSPN at the time of viral infection. In the current study, we found that poly IC treatment induces expression of Fkn in MCs in a time- and dose-dependent manner. The innate immune system recognized viral infection by detecting viral components, and TLR3, RIG-I, and MDA5 serve as pattern-recognition sensors for viral double-stranded RNA (20-23). Of note, treatment of cells with poly IC/cationic lipid complex did not induce glomerular Fkn expression, although it markedly induced RIG-I expression. Furthermore, poly IC treatment clearly increased both the secreted and cell-associated Fkn protein, but this was not the case with poly IC/cationic lipid treatment. These results suggest that poly IC-induced expression of Fkn in MCs does not depend on cytosolic RNA recognition sensors such as RIG-I but may depend on the extracellular sensor TLR3, suggesting that mesangial Fkn expression is tightly regulated. Moreover, poly IC treatment induced neither the expression of TNF- $\alpha$ nor that of IL- $1 \beta$ mRNA in the current study, although proinflammatory cytokines are known as potent inducers of Fkn, suggesting that neither TNF- $\alpha$ nor IL- $1 \beta$ may be directly involved in poly IC-induced Fkn expression in MCs.

We found that siRNA-mediated knockdown of TLR3 suppressed poly IC-induced Fkn expression. However, siRNAmediated knockdown of neither RIG-I nor MDA5 affected poly IC-induced expression of Fkn in the MCs. These results indicate that TLR3 works as a dominant sensor for this reaction. We then examined this signaling pathway in more detail. It has been reported that recognition of viral double-stranded RNA by TLR3 activates IRF3, NF- $\kappa B$ p65, and AP-1, which is followed by transcription of the target genes. Because we previously found that poly IC-induced signaling via TLR3 in MCs results in the induction of IFN- $\beta$, and newly synthesized IFN- $\beta$ induces the expression of ISGs $(14,15)$, we examined 


\section{Articles | Aizawa-Yashiro et al.}

Table 1. Oligonucleotide primers used in real-time quantitative PCR

\begin{tabular}{|c|c|c|c|c|}
\hline cDNA & Primers & Annealing temperature $\left({ }^{\circ} \mathrm{C}\right)$ & Cycles & Product (bp) \\
\hline \multirow[t]{2}{*}{ Fractalkine } & F: 5'-GACCCCTAAGGCTGAGGAAC-3' & 55 & 40 & 205 \\
\hline & R: 5'-CTCTCCTGCCATCTTTCGAG-3' & & & \\
\hline & R: 5'-TGGCTTGGGATGTGGTCTACTC-3' & & & \\
\hline ISG15 & F: 5'-GGCTGGGACCTGACGGTGAAG-3' & 55 & 40 & 570 \\
\hline \multirow[t]{2}{*}{ GAPDH } & F: 5'-GCACCGTCAAGGCTGAGAAC-3' & 55 & 40 & 142 \\
\hline & R: 5'-ATGGTGGTGAAGACGCCAGT-3' & & & \\
\hline
\end{tabular}

CDNA, complementary DNA; GAPDH, glyceraldehyde-3-phosphate dehydrogenase; ISG15, interferon-stimulated gene 15; RIG-I, retinoic acid-inducible gene-I.

Table 2. Oligonucleotide primers used in RT-PCR

\begin{tabular}{|c|c|c|c|c|}
\hline CDNA & Primers & Annealing temperature $\left({ }^{\circ} \mathrm{C}\right)$ & Cycles & Product (bp) \\
\hline \multirow[t]{2}{*}{ TNF-a } & F: 5'-GGCAGTCAGATCATCTTCTCGGAA-3' & 55 & 30 & 850 \\
\hline & R: 5'-GAAGGCCTAAGGTCCACTTGTGT-3' & & & \\
\hline IL-1 $\beta$ & R: 5'-ACACAAATTGCATGGTGAAGTCAGTT-3' & & & \\
\hline GAPDH & F: 5'-CCACCCATGGCAAATTCCATGGCA-3' & 55 & 30 & 598 \\
\hline
\end{tabular}

CDNA, complementary DNA; GAPDH, glyceraldehyde-3-phosphate dehydrogenase; IL, interleukin; RT-PCR, reverse transcription-PCR; TNF, tumor necrosis factor.

Table 3. Clinical characteristics of nine patients

\begin{tabular}{|c|c|c|c|c|c|c|c|}
\hline Case & Clinical diagnosis & Gender & $\begin{array}{l}\text { Age at biopsy } \\
\text { (years) }\end{array}$ & Renal pathology & $\begin{array}{l}\text { Glomerular } \\
\text { sclerosis or } \\
\text { crescent (\%) }\end{array}$ & $\begin{array}{l}\text { U-prot at biopsy } \\
\text { (g/day) }\end{array}$ & $\begin{array}{l}\text { Estimated GFR } \\
\left(\mathrm{ml} / \mathrm{min} / 1.73 \mathrm{~m}^{2}\right)\end{array}$ \\
\hline$A$ & $\mathrm{LN}+\mathrm{NS}$ & Male & 19 & ISN/RPS class IV-G (A/C $)^{\mathrm{b}}$ & 56.3 & 5.2 & 63.5 \\
\hline C & Nutcracker syndrome & Male & 14 & Normal glomeruli & 0 & 0.3 & 128.4 \\
\hline D & $\lg A N$ & Male & 11 & $\mathrm{Al}=4, \mathrm{Cl}=4^{\mathrm{c}}$ & 25.9 & 0.3 & 120.6 \\
\hline $\mathrm{F}$ & $\lg A N$ & Female & 8 & $\mathrm{Al}=2, \mathrm{Cl}=2^{\mathrm{c}}$ & 0 & 0.3 & 113.7 \\
\hline G & $\mathrm{HSPN}+\mathrm{NS}$ & Male & 7 & ISKDC grade III $\mathrm{b}^{\mathrm{d}}$ & 29 & 4.2 & 119.0 \\
\hline $\mathrm{H}$ & HSPN & Male & 5 & ISKDC grade IIIa ${ }^{d}$ & 19.2 & 2.0 & 117.0 \\
\hline । & HSPN & Female & 8 & ISKDC grade $\|^{d}$ & 0 & 0.7 & 130.3 \\
\hline
\end{tabular}

Al, activity index; Cl, chronicity index (Al and CI were described by Andreoli and Bergstein) (28); GFR, glomerular filtration rate; HSPN, Henoch-Schölein purpura nephritis; IgAN, IgA nephropathy; ISKDC, International Study of Kidney Disease in Children; ISN/RPS, International Society of Nephrology/Renal Pathology Society; LN, lupus nephritis; MCNS, minimalchange nephrotic syndrome; NS, nephrotic syndrome; U-prot, urinary protein excretion.

aThe rate of the number of glomeruli including the sclerosis or crescent in the total number of glomeruli observed. ${ }^{\circ}$ The disease activity of LN was classified by the ISN/RPS system; (A/C) means active associated with chronic lesions. IgAN was scored using the scoring system for childhood lgAN described by Andreoli and Bergstein (28). ${ }^{\mathrm{I}} \mathrm{HSPN}$ was classified by

ISKDC criteria.

the involvement of IRF3, NF- $\mathrm{B}$ p65, and ISG15 in this pathway. We observed that knockdown of IRF3, but not NF- $\mathrm{B}$ p65, inhibited poly IC-induced Fkn expression in MCs. On the other hand, knockdown of NF- $\mathrm{B}$ p65 clearly decreased the expression of IL- 8 induced by poly IC, suggesting that the expression of Fkn in poly IC-treated MCs is differently regulated from that of IL-8. In addition, pretreatment of cells using a blocking antibody against type I IFN receptor did not affect poly IC-induced mesangial Fkn expression, although it clearly inhibited poly IC-induced ISG15 expression. Furthermore, an
AP-1 inhibitor had no effect on poly IC-induced Fkn expression. These results suggest that poly IC-induced Fkn expression in MCs primarily depends on the TLR3/IRF3 signaling pathway, indicating that an activated TLR3/IRF3/Fkn signaling pathway may exist in MCs infected by some viruses. Recently, it has been reported that Fkn is not a target of IRF3dependent direct response genes in "embryo" fibroblasts from virally infected mice (23). In the current study, knockdown of IRF3 clearly decreased, but did not completely abolish, Fkn expression in poly IC-treated MCs. This discrepancy may be 
attributable to cell maturity, types, or species. Future detailed studies focused on this issue are needed. Because viral infection is reported to induce matrix metalloproteinase and growth factors in MCs, resulting in the development of glomerular sclerosis (13), a cross-talk of Fkn and those proinflammatory factors induced by viral infection may have an important role in the exacerbation and progression of glomerular diseases.

In human renal diseases, it has previously been reported that Fkn expression increases in glomerular or tubulointerstitial areas in biopsy specimens from patients with vasculitic glomerulonephritis and acute renal allograft rejection, but this was not observed in noninflammatory glomerulonephritis or normal tissue (24). Moreover, it has been reported that disease activity and proliferative lesions in patients with proliferative LN (International Society of Nephrology/Renal Pathology Society (ISN/RPS) class III and IV) are associated with glomerular Fkn expression and intrarenal recruitment of $\mathrm{CD} 16^{+}$monocytes. Steroid treatment resulted in a tendency to decrease both glomerular Fkn expression and $\mathrm{CD} 16^{+}$monocyte infiltration (8). However, the importance of glomerular Fkn expression in human renal diseases remains to be elucidated. RNA virus infections exacerbate the disease activity of IgAN (12). Because HSPN results from the inflammatory reactions due to deposition of mesangial IgAs such as IgAN (25), we next conducted a pilot immunofluorescence study using renal biopsy specimens obtained from patients with IgAN and HSPN to examine the involvement of glomerular Fkn expression. In the current study, we included nine stored renal biopsy specimens in good condition. Glomerular immunoreactivity for Fkn was detectable with an intense granular pattern in the mesangial area and capillary loop distribution in two patients with active IgAN and two patients with crescentic HSPN (ISKDC grade IIIa and IIIb), as well as a patient with proliferative LN of the ISN/RPS class IV-G (active lesion combined with chronic lesion). Furthermore, the immunostaining intensity was weak in patients with inactive IgAN and noncrescentic HSPN (ISKDC grade II). In cases with nonproliferative glomerular disease, such as minimal-change nephrotic syndrome, and noninflammatory renal tissue, such as that seen in nutcracker syndrome, immunofluorescence staining was absent in the glomeruli. In this pilot study, although we did not examine intrarenal infiltrating cells expressing the Fkn receptor, glomerular Fkn expression in patients with IgAN and HSPN may be related to the severity of intrarenal inflammation as postulated in a recent report describing LN (8). These preliminary observations suggest that even in some patients with IgAN and HSPN, activation of the virus infection-induced TLR3/IRF3/ Fkn pathway may be involved in the development of glomerular inflammation, although this theory remains speculative. These clinical and experimental observations, as well as those of previous reports examining patients with proliferative LN (8), suggest that Fkn may be a candidate therapeutic target for selected patients with proliferative glomerulonephritis.

We conclude that poly IC induces the expression of Fkn in cultured normal human MCs. TLR3 and IRF3 are involved in poly IC-induced Fkn expression. We also demonstrated increased glomerular Fkn expression in biopsy specimens from some patients with active IgAN and crescentic HSPN. Fkn may be involved in the pathogenesis of these inflammatory glomerulonephritis conditions, as well as in the aggravation of these diseases induced by viral infections. Thus, we believe that the involvement of the TLR3/IRF3/Fkn pathway in poly IC signaling in MCs may be important in immune and inflammatory reactions against viral infections in MCs. However, we also think that this study remains speculative. To resolve this, future studies using Fkn-deficient mice are needed.

\section{METHODS}

This study was approved by the ethics committee of Hirosaki University Graduate School of Medicine, Hirosaki, Japan.

\section{Reagents}

Poly IC and antihuman Fkn rabbit antibody were from Sigma (St. Louis, MO). RNeasy total RNA isolation kit; siRNAs against TLR3 (SI02655156), RIG-I (SI03019646), MDA5 (SI03649037), and IFN regulatory factor 3 (SI03117359); and nonsilencing negative control siRNA (1027281) were from Qiagen (Hilden, Germany). Primer oligo(dT) ${ }_{12-18}$, dNTP mix, Moloney murine leukemia virus reverse transcriptase, and Lipofectamine RNAi MAX were purchased from Invitrogen (Carlsbad, CA). SsoFast EvaGreen Supermix was from Bio-Rad (Hercules, CA). Oligonucleotide primers for PCR were custom synthesized by Greiner (Atsugi, Japan). An antibody against TLR3 and fluorescein isothiocyanate-conjugated secondary antirabbit antibody were from Santa Cruz Biotechnology (Santa Cruz, CA). Antibodies against anti-IRF3 (18781) and MDA5 (29020) were from Immuno-Biological Laboratories (Takasaki, Japan). Anti-RIG-I antibody was obtained according to previously described methods (26). The monoclonal antibody against type I IFN receptor was from Calbiochem (La Jolla, CA). siRNA against NF- $\kappa B$ p65 and an anti-p65 rabbit monoclonal antibody (C22B4) were from Cell Signaling Technologies (Danvers, MA). Enzyme-linked immunosorbent assay kits for Fkn and IL-8, and an AP-1 inhibitor (SR11302), were from R\&D Systems (Minneapolis, MN).

\section{Cells}

MCs and culture media were purchased from Lonza (Walkersville, $\mathrm{MD}$ ), and the cells were cultured according to the manufacturer's protocol. The cultured medium was supplemented with $5 \%$ fetal bovine serum, $50 \mu \mathrm{g} / \mathrm{ml}$ gentamicin, and $50 \mathrm{ng} / \mathrm{ml}$ amphotericin B. Poly IC was dissolved in phosphate-buffered saline, $\mathrm{pH} 7.4$, and the cells were treated with $2-50 \mu \mathrm{g} / \mathrm{ml}$ poly IC for up to $48 \mathrm{~h}(14,15,27)$. Furthermore, the cells were treated with $1 \mathrm{ng} / \mathrm{ml}$ poly IC in combination with cationic lipid (Lipofectamine RNAi MAX). In a pilot experiment, we found that treatment of more than $10 \mathrm{ng} / \mathrm{ml}$ poly IC/cationic lipid complex was cytotoxic (data not shown). In siRNA experiments, cells were transfected using nonsilencing control siRNA or siRNA against TLR3, RIG-I, MDA5, IRF3 or NF- $\mathrm{kB}$ p65 by using a Lipofectamine RNAi MAX reagent. One day before transfection, the culture medium was replaced with a medium not containing antibiotics. In the experiment using SR11302, an AP-1 inhibitor, the cells were pretreated with $5 \mu \mathrm{mol} / 1$ SR11302 $1 \mathrm{~h}$ before treatment with poly IC.

\section{Real-Time PCR Quantitative Analyses}

Total RNA was extracted from cells using an RNeasy RNA extraction kit. Single-stranded complementary DNA was synthesized from $1 \mu \mathrm{g}$ of total RNA using oligo(dT) ${ }_{12-18}$ primer and Moloney murine leukemia virus reverse transcriptase. The complementary DNA for Fkn, RIG-I, TNF- $\alpha$, IL-1 $\beta$, ISG15, and glyceraldehyde-3-phosphate dehydrogenase was amplified using SsoFast EvaGreen Supermix. The primers used are shown in Tables 1 and 2.

\section{Enzyme-Linked Immunosorbent Assay for Fkn}

The secreted form of Fkn and IL-8 concentrations in cell-conditioned medium were measured using enzyme-linked immunosorbent assay kits. The concentration of the cell-associated form of Fkn was also 
measured in lysates obtained from poly IC-treated or poly IC/cationic lipid complex-treated cells.

\section{Western Blot Analysis}

Western blot analysis was performed as described in previous reports $(14,27)$. Briefly, cells were washed twice with $20 \mathrm{mmol} / \mathrm{l}$ cold phosphate-buffered saline and lysed with Laemmli's lysis buffer. The lysate was subjected to electrophoresis on a 7.5 or $10 \%$ polyacrylamide gel; the proteins were subsequently transferred to a polyvinylidene difluoride membrane. TLR3, RIG-I, MDA5, IRF3, and NF-kB p65 were detected using antibodies against TLR3 (1:200 dilution), RIG-I (1:10,000 dilution), MDA5 (1:100 dilution), IRF3 (1:200 dilution), and NF-кB p65 (1:1,000 dilution), respectively, and subsequently with horseradish peroxidase-labeled antirabbit immunoglobulin G. Immunodetection was performed using a chemiluminescent substrate.

\section{Immunofluorescence Staining of Fkn}

Snap-frozen sections, stored in good condition, from patients with IgAN $(n=3)$, HSPN $(n=3)$, LN (served as a positive control, $n=1)$, minimalchange nephrotic syndrome $(n=1)$, and nutcracker syndrome (served as a noninflammatory control, $n=1$ ) were stained for Fkn. In these patients, renal biopsy specimens were obtained as a routine diagnostic clinical examination before the study and were subsequently stored. Informed consent was obtained from all study participants. The clinical characteristics of the nine study patients are shown in Table 3. The AI and CI of IgAN were scored semiquantitatively by using the scoring system for childhood IgA nephropathy described by Andreoli and Bergstein (28), and HSPN was classified on the basis of the ISKDC criteria. LN disease activity was classified according to the ISN/RPS system.

The optimal cutting temperature-embedded tissue specimens were cut into 5 - $\mu \mathrm{m}$-thick sections using a cryostat, briefly fixed in cold acetone, and air-dried; the slides were then washed in phosphate-buffered saline immediately before immunohistochemical analysis. Anti-Fkn antibody was added at a concentration of 1:200. After incubation for $24 \mathrm{~h}$ at $4{ }^{\circ} \mathrm{C}$ and several washes in phosphate-buffered saline, the slides were incubated sequentially with fluorescein isothiocyanate-conjugated secondary antibodies at a concentration of $1: 40$ for $30 \mathrm{~min}$ at $37^{\circ} \mathrm{C}$. The microscope was an Olympus IX70 model (Olympus, Tokyo, Japan), and the magnification was $\times 200$. Unfortunately, we did not examine intrarenal infiltrating cells expressing the Fkn receptor in the specimen because the stored frozen sections were insufficient for the study.

\section{Statistical Analysis}

Statistical significance was evaluated using the paired $t$-test.

\section{ACKNOWLEDGMENTS}

The authors thank Kumiko Munakata, Miho Ono, Michiko Nakata, and Ai Yamamoto for their help.

\section{STATEMENT OF FINANCIAL SUPPORT}

This work was supported by a Grant-in-Aid for Science from the Ministry of Education, Culture, Sports, Science and Technology of Japan (22591175 to H.T. and T.I.) and the Karoji Memorial Fund for Medical Research in Hirosaki University (to T.I. and K.T.).

Disclosure: The authors declared no conflict of interest.

\section{REFERENCES}

1. Rovin BH, Phan LT. Chemotactic factors and renal inflammation. Am J Kidney Dis 1998;31:1065-84.

2. Segerer S, Nelson PJ, Schlöndorff D. Chemokines, chemokine receptors, and renal disease: from basic science to pathophysiologic and therapeutic studies. J Am Soc Nephrol 2000;11:152-76.

3. Anders HJ, Vielhauer V, Schlöndorff D. Chemokines and chemokine receptors are involved in the resolution or progression of renal disease. Kidney Int 2003;63:401-15.

4. Bazan JF, Bacon KB, Hardiman G, et al. A new class of membrane-bound chemokine with a CX3C motif. Nature 1997;385:640-4.

5. Sukkar MB, Issa R, Xie S, Oltmanns U, Newton R, Chung KF. Fractalkine/ CX3CL1 production by human airway smooth muscle cells: induction by IFN- $\gamma$ and TNF- $\alpha$ and regulation by TGF- $\beta$ and corticosteroids. Am J Physiol Lung Cell Mol Physiol 2004;287:L1230-40.
6. Yoshida H, Imaizumi T, Fujimoto K, et al. Synergistic stimulation, by tumor necrosis factor- $\alpha$ and interferon- $\gamma$, of fractalkine expression in human astrocytes. Neurosci Lett 2001;303:132-6.

7. Ito $\mathrm{Y}$, Kawachi H, Morioka Y, et al. Fractalkine expression and the recruitment of CX3CR1+ cells in the prolonged mesangial proliferative glomerulonephritis. Kidney Int 2002;61:2044-57.

8. Yoshimoto S, Nakatani K, Iwano M, et al. Elevated levels of fractalkine expression and accumulation of CD16+ monocytes in glomeruli of active lupus nephritis. Am J Kidney Dis 2007;50:47-58.

9. Nakatani K, Yoshimoto S, Iwano M, et al. Fractalkine expression and CD16+ monocyte accumulation in glomerular lesions: association with their severity and diversity in lupus models. Am J Physiol Renal Physiol 2010;299:F207-16.

10. Inoue A, Hasegawa $\mathrm{H}, \mathrm{Kohno} \mathrm{M}$, et al. Antagonist of fractalkine (CX3CL1) delays the initiation and ameliorates the progression of lupus nephritis in MRL/lpr mice. Arthritis Rheum 2005;52:1522-33.

11. Segerer S, Hughes E, Hudkins KL, Mack M, Goodpaster T, Alpers CE. Expression of the fractalkine receptor (CX3CR1) in human kidney diseases. Kidney Int 2002;62:488-95.

12. Lai AS, Lai KN. Viral nephropathy. Nat Clin Pract Nephrol 2006;2:254-62.

13. Wörnle M, Roeder M, Sauter M, Ribeiro A. Role of matrix metalloproteinases in viral-associated glomerulonephritis. Nephrol Dial Transplant 2009;24:1113-21.

14. Imaizumi $\mathrm{T}$, Tanaka $\mathrm{H}$, Matsumiya $\mathrm{T}$, et al. Retinoic acid-inducible gene-I is induced by double-stranded RNA and regulates the expression of CC chemokine ligand (CCL) 5 in human mesangial cells. Nephrol Dial Transplant 2010;25:3534-9.

15. Imaizumi $\mathrm{T}$, Tanaka $\mathrm{H}$, Mechti $\mathrm{N}$, et al. Polyinosinic-polycytidylic acid induces the expression of interferon-stimulated gene 20 in mesangial cells. Nephron Exp Nephrol 2011;119:e40-8.

16. Patole PS, Gröne HJ, Segerer S, et al. Viral double-stranded RNA aggravates lupus nephritis through Toll-like receptor 3 on glomerular mesangial cells and antigen-presenting cells. J Am Soc Nephrol 2005;16:1326-38.

17. Flür K, Allam R, Zecher D, et al. Viral RNA induces type I interferondependent cytokine release and cell death in mesangial cells via melanomadifferentiation-associated gene-5: implications for viral infection-associated glomerulonephritis. Am J Pathol 2009;175:2014-22.

18. Chen YM, Lin SL, Chen CW, Chiang WC, Tsai TJ, Hsieh BS. Tumor necrosis factor-alpha stimulates fractalkine production by mesangial cells and regulates monocyte transmigration: down-regulation by cAMP. Kidney Int 2003;63:474-86.

19. Wu SH, Lu C, Dong L, Chen ZQ. Signal transduction involved in CTGFinduced production of chemokines in mesangial cells. Growth Factors 2008;26:192-200.

20. Kato H, Takeuchi O, Sato S, et al. Differential roles of MDA5 and RIG-I helicases in the recognition of RNA viruses. Nature 2006;441:101-5.

21. Yoneyama M, Kikuchi M, Natsukawa T, et al. The RNA helicase RIG-I has an essential function in double-stranded RNA-induced innate antiviral responses. Nat Immunol 2004;5:730-7.

22. Alexopoulou L, Holt AC, Medzhitov R, Flavell RA. Recognition of doublestranded RNA and activation of NF-kappaB by Toll-like receptor 3. Nature 2001;413:732-8.

23. Anderson J, VanScoy S, Cheng TF, Gomez D, Reich NC. IRF-3-dependent and augmented target genes during viral infection. Genes Immun 2008;9:168-75.

24. Cockwell P, Chakravorty SJ, Girdlestone J, Savage CO. Fractalkine expression in human renal inflammation. J Pathol 2002;196:85-90.

25. Davin JC, Weening JJ. Henoch-Schönlein purpura nephritis: an update. Eur J Pediatr 2001;160:689-95.

26. Imaizumi T, Tanaka H, Tajima A, et al. IFN- $\gamma$ and TNF- $\alpha$ synergistically induce microRNA-155 which regulates TAB2/IP-10 expression in human mesangial cells. Am J Nephrol 2010;32:462-8.

27. Imaizumi $\mathrm{T}$, Aratani $\mathrm{S}$, Nakajima $\mathrm{T}$, et al. Retinoic acid-inducible gene-I is induced in endothelial cells by LPS and regulates expression of COX-2. Biochem Biophys Res Commun 2002;292:274-9.

28. Andreoli SP, Bergstein JM. Treatment of severe IgA nephropathy in children. Pediatr Nephrol 1989;3:248-53. 